\title{
Three-component bioorthogonal reactions on cellular DNA and RNA
}

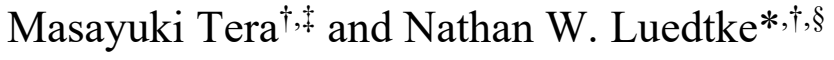 \\ †Department of Chemistry, University of Zurich, Winterthurerstrasse 190, 8057 Zurich, \\ Switzerland. \\ Current address: Department of Biotechnology and Life Sciences, Tokyo University of \\ Agriculture and Technology, 2-24-16 Nakacho, Koganei, Tokyo 184-8588, Japan. \\ ${ }^{\S}$ Current address: Department of Chemistry, McGill University, 801 Sherbrooke St. West \\ Montréal, Québec H3A 0B8, Canada. e-mail: nathan.luedtke@mcgill.ca
}

\section{Electronic Supporting Information}

1. General synthetic methods and reagents. AmdU triphosphate ${ }^{1}, P O M-A m d U^{2}$ and DiMOC $^{3}$ were synthesized as previously reported. All other reagents were obtained in the highest commercial grades from Sigma Aldrich, TCI Europe, Merck, or Fluorochem without further purification. All non-aqueous reactions were conducted under nitrogen atmosphere using anhydrous solvents. NMR spectra were measured with a Bruker AVIII-400 or 500 (400 $\mathrm{MHz}$ for ${ }^{1} \mathrm{H}, 100 \mathrm{MHz}$ for ${ }^{13} \mathrm{C}$ or $500 \mathrm{MHz}$ for ${ }^{1} \mathrm{H}, 125 \mathrm{MHz}$ for $\left.{ }^{13} \mathrm{C}\right)$. Chemical shifts $(\delta)$ are given in parts per million (ppm) and are reported relative to residual solvent peaks: $\mathrm{CDCl}_{3}(\delta \mathrm{H}$ $7.26, \delta \mathrm{C} 77.0 \mathrm{ppm}), \mathrm{CD}_{3} \mathrm{CN}(\delta \mathrm{H} 1.94, \delta \mathrm{C} 1.32 \mathrm{ppm})$. Coupling constants $(J)$ are given in Hertz $(\mathrm{Hz}) .{ }^{13} \mathrm{C}$-spectra were recorded broadband proton decoupled. High-resolution electrospray mass spectra were recorded on a Bruker maXis QTOF-MS instrument. Electrospray Ionization (ESI) mass spectra were obtained on a quadrupole ion trap instrument equipped with an atmospheric pressure ion (API) source. Masses are given as $\mathrm{m} / \mathrm{z}$.

\section{General experimental procedures for biochemistry experiments.}

All oligonucleotides were purchased from Sigma-Aldrich (HPLC purification grade) and used without further purification. DNA polymerase I, large (Klenow) fragment (M0120), dNTP solutions were purchased from New England BioLabs. All other reagents were obtained in the molecular biology grades from Sigma-Aldrich, Thermo Fischer Scientific, TriLink, or GE Healthcare. Sep-pak C18 reverse phase columns were purchased from Waters and used according to the manufacturer's instructions. Gel-electrophoresis was carried in a vertical 
electrophoresis unit (Bio-Rad, Model: Mini-PROTEAN II cell). 15-well combs were used for all gel analyzes. The gels were scanned by Typhoon FLA 9500 (GE Healthcare) and analyzed by ImageJ $1.47 \mathrm{c}$ (National Institutes of Health, USA). MALDI-MS analyses (for the synthesized oligonucleotides) were performed on a Bruker Autoflex time-of-flight mass spectrometer (Bruker Daltonics, Bremen, Germany) equipped with a $337 \mathrm{~nm} \mathrm{~N}$ laser in the reflectron (or in the linear) mode. Confocal images were acquired at the Center for Microscopy and Image Analysis at the University of Zurich on a CLSM Leica SP5 Mid UV-VIS (63x oil immersion objective, NA 1.4) confocal microscope. Image analysis was performed using ImageJ $1.47 \mathrm{c}$ (National Institutes of Health, USA).

Table S1. Purchased oligonucleotides

Name Sequences (5'-3')

P1 FAM-TCGTCTCTTGTCTG

T1 TGCTGCGTTGTGTACAGACAAGAGACGATTTTTTTTTTTT

Table S2. Oligonucleotide containing AmdU " $\underline{T}$ ” prepared by primer extension

\begin{tabular}{llrr}
\hline Name & Sequences (5'-3') & calcd MS & Found \\
\hline FAM- & & \\
oligo1 & TCGTCTCTTGTCTGTACACAACGCAGCA & 8613.7 & 8615.1
\end{tabular}

\section{Primer extension and purification of AmdU-containing oligonucleotides}

For each reaction, $39 \mu \mathrm{M}$ of DNA primer and $30 \mu \mathrm{M}$ of template were mixed in the presence of $1 \mathrm{x}$ PCR buffer (supplied with the enzyme; $50 \mathrm{mM} \mathrm{NaCl}, 10 \mathrm{mM} \mathrm{MgCl} 2,1 \mathrm{mM}$ DTT, 10 $\mathrm{mM}$ Tris- $\mathrm{HCl} \mathrm{pH} \mathrm{7.9)} \mathrm{and} \mathrm{heated} \mathrm{to} 95^{\circ} \mathrm{C}$ for $5 \mathrm{~min}$ and gradually cooled down to $25^{\circ} \mathrm{C}$. In a $30 \mu \mathrm{L}$ reaction, the annealed primer and template $(3 \mu \mathrm{M})$, were incubated in mixture containing $33 \mu \mathrm{M}$ of each nucleotide triphosphate (dATP, dGTP, dCTP, AmdU triphosphate), $1 \mathrm{U}$ of Klenow fragment (NewEngland BioLabs) at $25^{\circ} \mathrm{C}$ for $25 \mathrm{~min}$. The reaction mixtures were subjected to ethanol precipitation and quantified by UV absorbance. The precipitate was redissolved in $50 \mathrm{mM} \mathrm{NaCl}$ and annealed to form duplex. For the single-stranded azide-modified oligonucleotide, the primer extension product was purified by a preparative denaturing gel. After primer extension reaction, crude oligonucleotides were desalted by Sep-Pak C18 cartridges (Waters) and lyophilized. The dry samples were dissolved in $10 \mathrm{M}$ urea solution, heated to $85^{\circ} \mathrm{C}$ for $5 \mathrm{~min}$, and loaded onto a denaturing gel ( $7 \mathrm{M}$ urea, 19.3\% acrylamide, $0.7 \%$ bis-acrylamide in TBE buffer). For preparative gels, the desired bands were excised by a razor 
under the UV light from the gel and extracted by $100 \mathrm{mM}$ Triethylammonium acetate $(\mathrm{pH} 7.3)$ buffer for $5-12$ hours at $45{ }^{\circ} \mathrm{C}$. The eluted oligonucleotides were desalted by Sep-Pak C18 cartridges, dried over speed-vac, and re-dissolved in pure water. All resulting oligonucleotides' concentration were measured by UV-absorbance (Nano drop 2000c, Thermo Fischer Scientific).

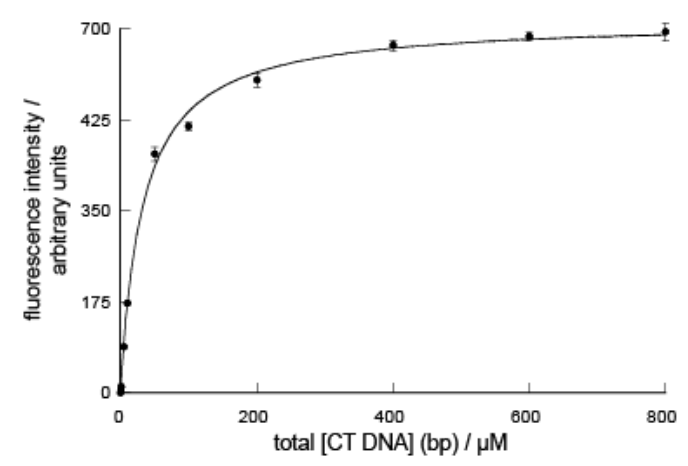

Figure S1. Fluorescence intensity of a $1 \mu \mathrm{M}$ solution of TO $\left(\lambda_{\mathrm{ex}}=485 \mathrm{~nm}, \lambda_{\mathrm{em}}=538 \mathrm{~nm}\right)$ upon addition of variable concentrations of calf thymus (CT) DNA. Averages and standard deviations of three independent replicates are shown.

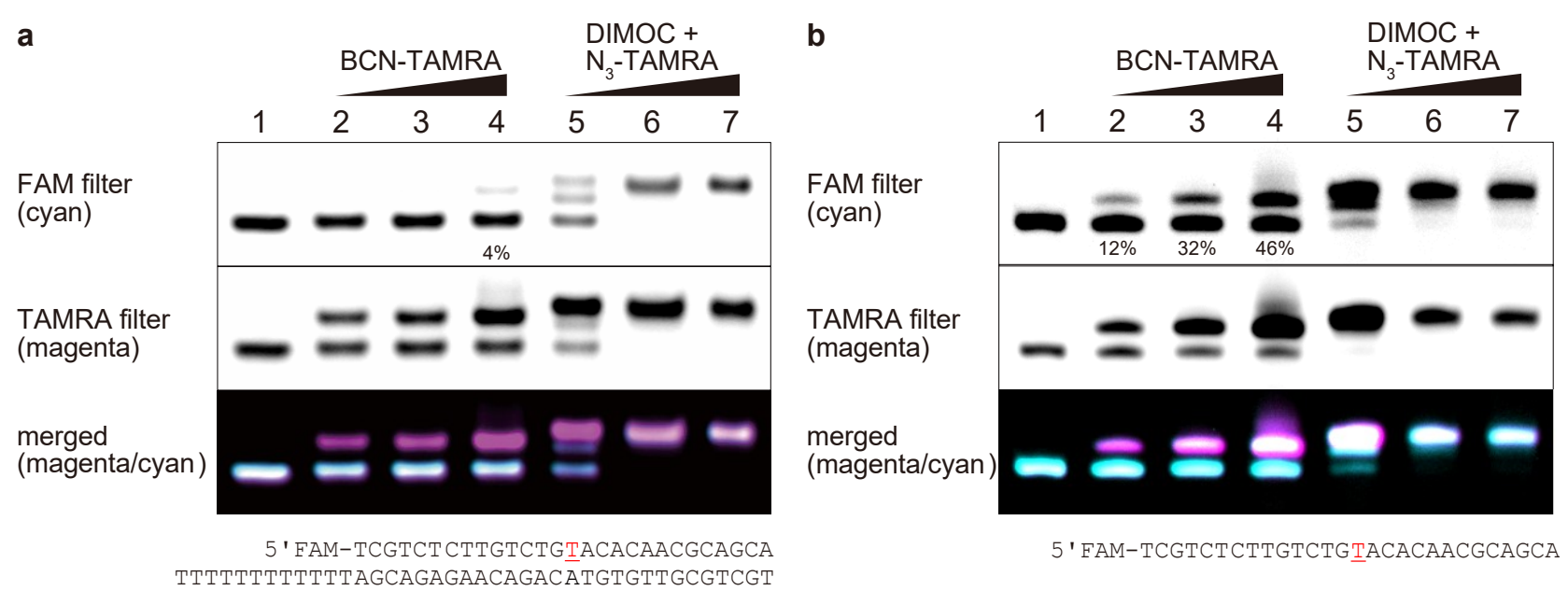

Figure S2. DPAGE analysis of reactions between duplex (a) or single-stranded DNA (b) containing a single AmdU residue "T" with BCN-TAMRA $(0.5-25 \mu \mathrm{M}, 20 \mathrm{~min})$ or DiMOC/N 3 -TAMRA $(0.5-25 \mu \mathrm{M}, 10 \mathrm{~min})$ in an aqueous buffer containing $200 \mathrm{mM} \mathrm{NaCl}$, $20 \mathrm{mM}$ (pH 5.2). Each sample was treated with BCN-TAMRA $(0.5-25 \mu \mathrm{M}, 20 \mathrm{~min})$ or DiMOC/N $\mathrm{N}_{3}$-TAMRA $(0.5-25 \mu \mathrm{M}, 10 \mathrm{~min})$ at rt. Following DPAGE, the gels were visualized by FAM- and TAMRA-filters and the merged image was generated by Image J. 


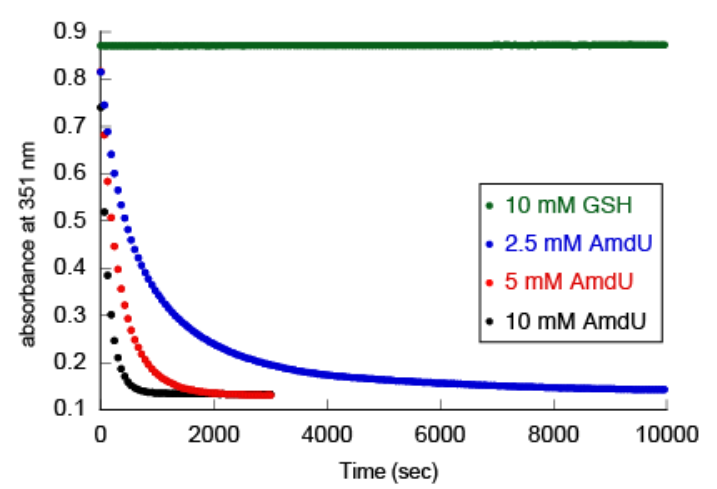

Figure S3. Normalized changes in DiMOC absorbance $(351 \mathrm{~nm})$ versus time in the presence of various concentrations of 5-azidomethyl-2'-deoxyuridine (AmdU) or glutathione (GSH). The initial reactions contained $0.6 \mathrm{mM}$ DiMOC in an aqueous buffer containing $50 \mathrm{mM}$ $\mathrm{NaOAc}(\mathrm{pH} 5.2)$ and $0.1 \% \mathrm{DMSO}$ at $25^{\circ} \mathrm{C}$.

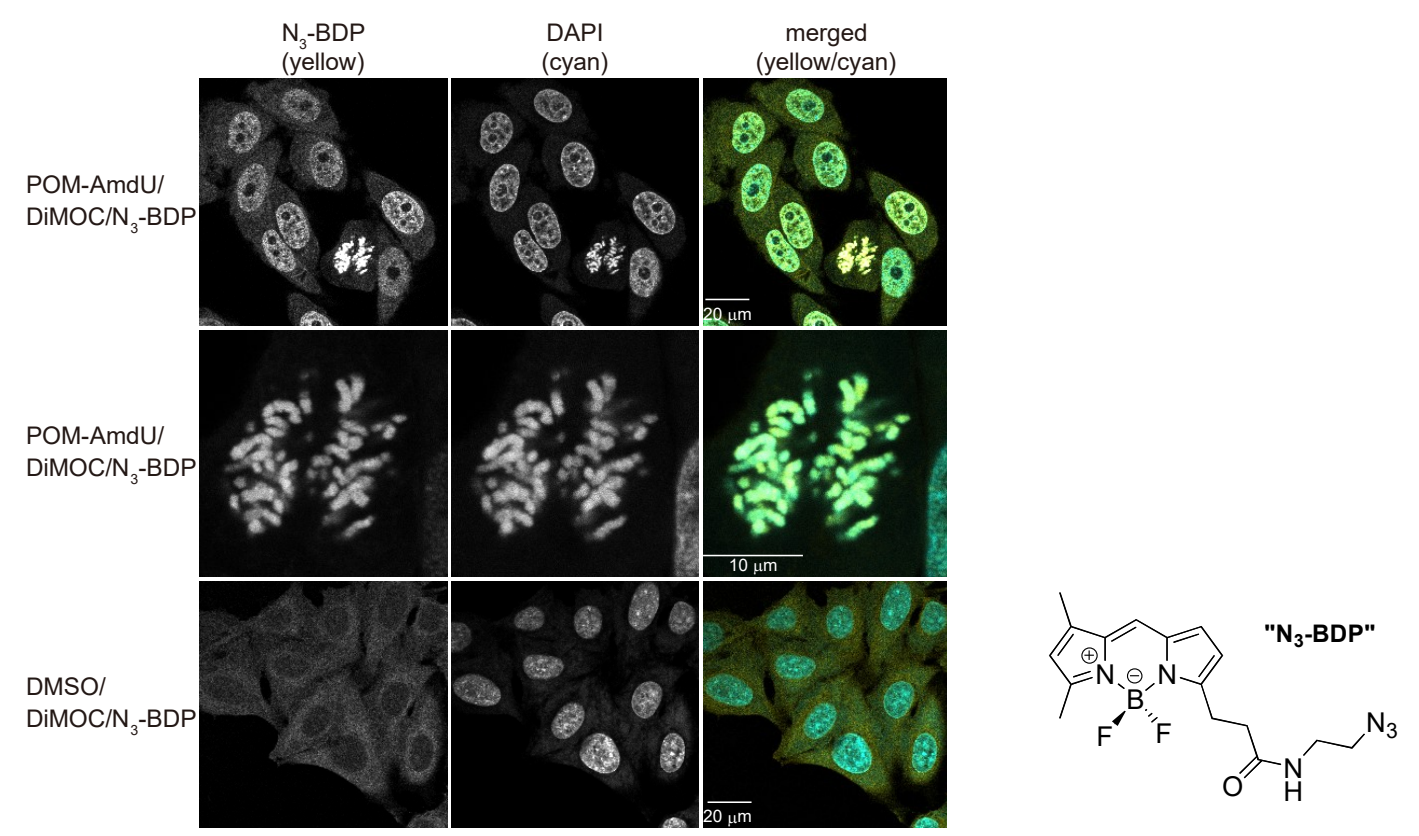

Figure S4. AmdU-DiMOC reactions conducted in living cells, fixed and stained with a fluorescent azide. HeLa cells were treated with $30 \mu \mathrm{M}$ of POM-AmdU for $24 \mathrm{~h}$, washed with DMEM, and treated with $10 \mu \mathrm{M}$ of DiMOC for 3 hours. The cells were fixed with paraformaldehyde and permeabilized with $0.1 \%$ triton $\mathrm{X}-100$. The cells were then treated with $10 \mu \mathrm{M}$ of $\mathrm{N}_{3}$-BDP for $10 \mathrm{~min}$, washed and the nuclei non-covalently stained by DAPI (cyan). Negative controls received no POM-AmdU, but were otherwise treated identically. 


\section{REFERENCES}

(1) Ren, X., El-Sagheer, A. H., Brown, T. (2015) Azide and Trans-Cyclooctene DUTPs: Incorporation into DNA Probes and Fluorescent Click-Labelling. Analyst 140, 2671-2678.

(2) Tera, M., Glasauer, S. M. K., Luedtke, N. W. (2018) In Vivo Incorporation of Azide Groups into DNA by Using Membrane-Permeable Nucleotide Triesters. ChemBioChem 19, 1939-1943.

(3) Tera, M., Harati Taji, Z., Luedtke, N. W. (2018) Intercalation-Enhanced "Click" Crosslinking of DNA. Angew. Chem., Int. Ed. 57, 15405-15409. 